\title{
Endocardial Invasion of Lung Cancer Undiagnosable before Autopsy
}
T. Mizuno ${ }^{a}$
K. Ishigami ${ }^{d}$
S. Yamada ${ }^{a, f}$
H. Tsuchiya ${ }^{a}$
C. Nakajima ${ }^{a}$
R. Sangen ${ }^{a}$
M. Fukushima ${ }^{\text {b }}$
H. Minato ${ }^{b}$
N. Nojimab
A. Saito ${ }^{c}$
N. Hayashic
H. Atsumi ${ }^{\mathrm{e}}$
T. Ito ${ }^{\mathrm{e}}$
M. Iguchi ${ }^{f}$
D. Usuda ${ }^{a}$
H. Okamura ${ }^{9}$
S. Urashima ${ }^{\mathrm{g}}$
M. Asano ${ }^{\text {h }}$
J. Kiyosawa ${ }^{\mathrm{h}}$
A. Fukudah
N. Takekoshi ${ }^{\text {h }}$
T. Kanda ${ }^{a}$

Departments of ${ }^{\mathrm{a} C o m m u n i t y ~ M e d i c i n e ~ a n d ~}{ }^{\mathrm{b}}$ Pathology and Laboratory Medicine, Kanazawa Medical University, Uchinada, Departments of ' Nephrology,

${ }^{\mathrm{d}}$ Geriatric Medicine, ${ }^{\mathrm{e}}$ Endocrinology, ${ }^{\mathrm{f}}$ Respiratory Medicine, ${ }^{\mathrm{g}}$ Gastroenterology, and

${ }^{h}$ Cardiology, Himi Municipal Hospital, Kanazawa Medical University, Himi, Japan

\section{Key Words}

Endocardium · Lung cancer · Autopsy · Parathyroid hormone-related protein

\section{Abstract}

A 59-year-old male patient presented with left chest discomfort on admission. His medical history included encephalitis in childhood and his smoking history was 20 cigarettes per day for 40 years. A physical examination showed an anemic and edematous face with weak respiratory sounds in the left lung. The patient had elevated calcium levels and decreased hemoglobin and potassium. His parathyroid hormone-related protein level was elevated. Thoracic radiography showed cardiomegaly and computed tomography revealed a left lung mass with invasion of the heart and pleural effusion. Magnetic resonance imaging showed endocardial invasion of the tumor mass. Gallium-68 imaging revealed positive accumulation in the region surrounding the heart. No diagnoses were possible upon frequent cytology of his sputum and pleural effusion. The patient died from congestive heart failure with anoxia 38 days after admission. An autopsy revealed tumoral mass occlusion in the left main bronchus and tumoral invasion of the left atrium, left ventricle, and aorta. 


\section{Introduction}

Lung cancer is one of the most common primary tumors in cardiac metastasis [1]. This type of metastasis involves the pericardium or epicardium by direct invasion [2,3]. Herein, we report a case of a patient with lung cancer that invaded the left atrial endocardium, as diagnosed upon autopsy. This rare case is presented with literature precedents.

\section{Case Presentation}

A 59-year-old male patient presented with left chest discomfort on admission. His blood pressure was $86 / 54 \mathrm{~mm} \mathrm{Hg}$, with a pulse rate of 107 beats per minute and a body temperature of $35.7^{\circ} \mathrm{C}$. His medical history included encephalitis in childhood and his smoking history was 20 cigarettes per day for 40 years.

A physical examination showed an anemic and edematous face with weak respiratory sounds in the left lung. The patient had elevated C-reactive protein, leukocytes, and calcium levels, and decreased hemoglobin and potassium. Cancer markers such as carcinogenic antigen, neuron-specific enolase, squamous cell carcinoma, and cytokeratin-19 fragment were all elevated. The calcium level was $12.6 \mathrm{mg} / \mathrm{dl}$. His parathyroid hormone-related protein (PTHrP) level was elevated to $20.2 \mathrm{pmol} / \mathrm{l}$ (normal range: 0-1.1), and intact parathyroid hormone (PTH) was $2 \mathrm{pg} / \mathrm{ml}$ (normal range: 10-65).

Thoracic radiography showed cardiomegaly and computed tomography showed a left lung mass with invasion of the heart and pleural effusion (fig. 1). Magnetic resonance imaging (MRI) revealed a large mass surrounding the heart (fig. 2), and gallium-68 imaging showed positive accumulation in the region surrounding the heart (fig. 3). No malignant diagnoses were possible upon frequent cytology of his sputum and pleural effusion. Bronchoscopy proved impossible due to the critical risk to life. The patient died from congestive heart failure with anoxia 38 days after admission. An autopsy revealed tumoral mass occlusion in the left main bronchus and tumoral invasion of the left atrium, left ventricle, and aorta.

\section{Discussion}

We have presented a case of a lung cancer patient with tumoral invasion of the left atrial endocardium, as diagnosed upon autopsy. No malignant diagnoses were possible upon frequent cytology of his sputum and pleural effusion, probably due to tumoral occlusion of the left main bronchus.

The most common neoplasms associated with cardiac metastasis are lung cancer, lymphoma, breast cancer, leukemia, stomach cancer, and melanoma [2, 4]. In a series of autopsies, cardiac metastasis was present in $15-35 \%$ of all lung cancer patients [5]. The pericardium is the most commonly affected structure in the event of cardiac metastasis, with involvement being due to either direct invasion or lymphatic spread. The epicardium and myocardium are the next most common sites for metastasis, almost exclusively resulting from retrograde lymphatic spread. Around $8-10 \%$ of all lung cancers invade the heart, especially the left atrium [6].

A comprehensive evaluation of cardiac mass, including the use of other imaging modalities (such as computed tomography or MRI), along with an assessment of the patient's clinical history and other laboratory findings, may be needed for optimal clinical decision- 
making. Cardiac MRI is a particularly useful imaging modality for soft-tissue characterization and is frequently used to localize and analyze the morphological appearance and infiltration of cardiac and juxta-cardiac structures [7].

Hypercalcemia is commonly observed in patients with cancer and bone metastases. Humoral hypercalcemia of malignancy is defined as hypercalcemia associated with a malignant tumor and is induced by the production of a hypercalcemic factor by tumor cells. PTHrP rather than PTH is currently considered its mediator [8]. Indeed, PTHrP levels are elevated in the serum of most patients with squamous cell carcinoma [9], which produces biochemical features of hyperparathyroidism upon binding to a common receptor for PTH and PTHrP [10]. Despite the structural homology between the amino termini of the two hormones, immunoradiometric assays for PTH can differ between patients with humoral hypercalcemia of malignancy and those with primary hyperparathyroidism [11]. In such cases, hypercalcemia is secondary to direct bone resorption and release of calcium and phosphorus into the bloodstream.

In the present case, the diagnostic difficulty of lung cancer was due to a large mass occlusion in the left main bronchus, which was detected upon autopsy. Since we were unable to perform a bronchoscopy due to severe dyspnea and anoxia, we could not establish an accurate diagnosis of lung cancer. According to the guidelines of the American College of Chest Physicians, a definitive diagnosis should be established by direct biopsy [12]. However, in this case, tumoral occlusion in the left main bronchus prevented cytological positivity with either a sputum or pleural effusion specimen. The cytological positivity of pleural effusion in lung cancer in the literature is $3.7-38.6 \%$ [13].

\section{Conclusion}

This is a rare case report of primary lung squamous cell carcinoma with invasion of the left atrial endocardium, as diagnosed upon autopsy with no cytological diagnosis when alive. MRI showed direct endocardial invasion by a large mass.

\section{Acknowledgement}

We would like to thank the patient and his family for their cooperation. This paper is not funded by any external source.

\section{Disclosure Statement}

The authors declare that they have no competing interests.

\section{References}

Klatt EC, Heitz DR: Cardiac metastases. Cancer 1990;65:1456-1459.

Reynen K, Köckeritz U, Strasser RH: Metastases to the heart. Ann Oncol 2004;15:375-381.

Kasai T, Kishi K, Kawabata M, et al: Cardiac metastasis from lung adenocarcinoma causing atrioventricular block and left ventricular outflow tract obstruction. Chest 2007;131:1569-1572.

4 Bussani R, De-Giorgio F, Abbate A, et al: Cardiac metastases. J Clin Pathol 2007;60:27-34.

5 Che GW, Liu LX, Zhang EY, et al: Left ventricular metastasis from a primary lung carcinoma. Chin Med J (Engl) 2007;120:2323-2324. 


\section{Case Reports in Oncology}

\begin{tabular}{l|l}
\hline Case Rep Oncol 2014;7:144-148 & \\
\hline DOI: $10.1159 / 000360394$ & $\begin{array}{l}\text { ○ 2014 S. Karger AG, Basel } \\
\text { www.karger.com/cro }\end{array}$ \\
\hline
\end{tabular}

Mizuno et al.: Endocardial Invasion of Lung Cancer Undiagnosable before Autopsy

6 Spaggiari L, D'Aiuto M, Veronesietal G: Extended pneumonectomy with partial resection of the left atrium, without cardiopulmonary bypass, for lung cancer. Ann Thorac Surg 2005;79:234-240.

7 Ma Q, Liu D, Liu P, et al: Extensive invasion of the left atrium by lung cancer. Ann Thorac Surg 2013;96:685687.

8 Suva LJ, Winslow GA, Wettenhall RE, et al: A parathyroid hormone-related protein implicated in malignant hypercalcemia: cloning and expression. Science 1987;237:893-896.

-9 Burtis WJ, Brady TG, Orloff JJ, et al: Immunochemical characterization of circulating parathyroid hormonerelated protein in patients with humoral hypercalcemia of cancer. N Engl J Med 1990;322:1106-1112.

-10 Orloff JJ, Wu TL, Stewart AF: Parathyroid hormone-like proteins: biochemical responses and receptor interactions. Endocr Rev 1989;10:476-495.

-11 Nussbaum SR, Zahradnik RJ, Lavigne JR, et al: Highly sensitive two-site immunoradiometric assay of parathyroid hormone in, and its clinical utility in evaluating patients with hypercalcemia. Clin Chem 1987;33:1364-1367.

12 Rivera MP, Mehta AC, Wahidi MM: Establishing the diagnosis of lung cancer: diagnosis and management of lung cancer, ed 3: American College of Chest Physicians evidence-based clinical practice guidelines. Chest 2013;143(5 suppl):e142S-165S.

13 Toufektzian L, Sepsas E, Drossos V, et al: Pleural lavage cytology: where do we stand? Lung Cancer 2014;83:14-22.

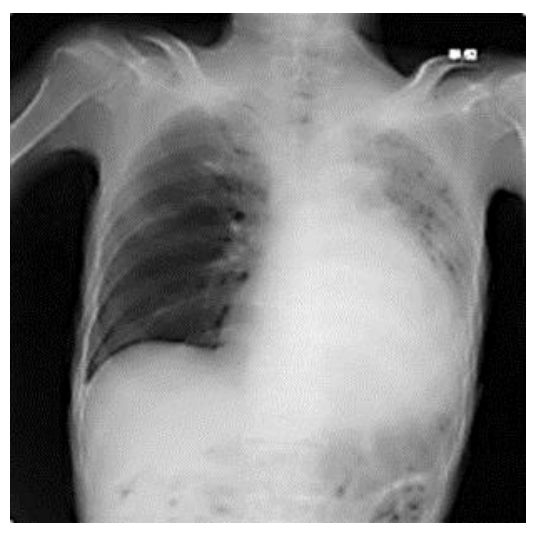

Fig. 1. Thoracic radiography showed cardiomegaly, and computed tomography revealed a left lung mass with invasion of the heart and pleural effusion.

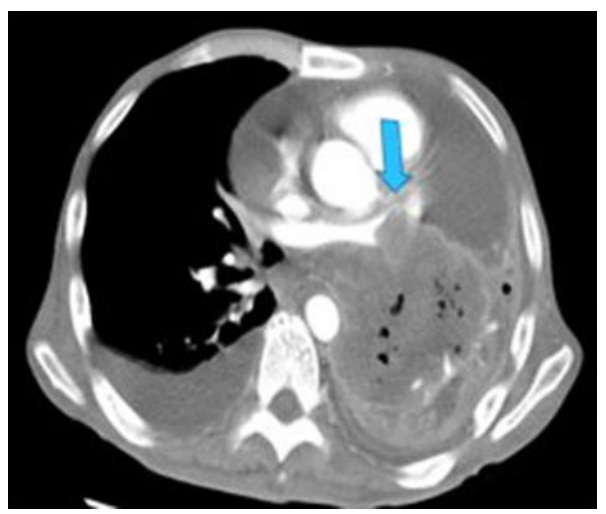

Fig. 2. MRI showed a large mass surrounding the heart. The arrow indicates endocardial invasion of a lesional mass. 


\section{Case Reports in Oncology}

Mizuno et al.: Endocardial Invasion of Lung Cancer Undiagnosable before Autopsy
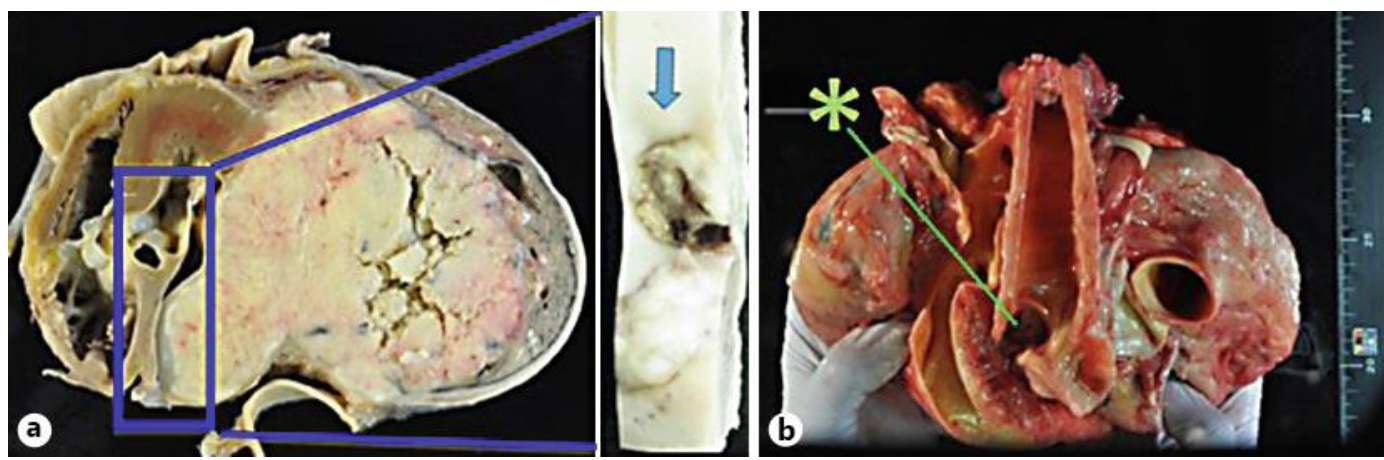

Fig. 3. a Autopsy showed left atrial invasion of lung cancer. The arrow indicates the endocardial mass at the atrial surface. $\mathbf{b}$ The asterisk shows mass occlusion in the left main bronchus, which may explain the difficulty in establishing a diagnosis by aspiration cytology. 